\title{
A PRÁTICA DO PIERCING EM ESTUDANTES UNIVERSITÁRIOS
}

\section{Samuel Santos Souza ${ }^{1}$; Matheus Cordeiro Fonseca ${ }^{2}$; Paula Cristina Pelli Paiva ${ }^{3}$; Haroldo Neves de Paiva ${ }^{4}$; Paulo Messias de Oliveira Filho ${ }^{5}$}

${ }^{1}$ Pós-Graduando em Imunologia e Parasitologia Básicas e Aplicadas, Universidade Federal de Mato Grosso (UFMT), Barra do Garças, Mato Grosso.

${ }^{2}$ Graduando em Odontologia, Universidade Federal dos Vales do Jequitinhonha e Mucuri (UFVJM), Diamantina, Minas Gerais.

${ }^{3,4,5}$ Doutor, Universidade Federal dos Vales do Jequitinhonha e Mucuri (UFVJM), Diamantina, Minas Gerais.

\section{DOI: 10.47094/IICNNESP.2021/59}

\begin{abstract}
RESUMO
O objetivo do presente estudo foi avaliar a prevalência e os fatores associados à prática do uso do piercing em universitários da área da saúde. Foi realizado um estudo transversal com amostra de conveniência de 190 universitários. Dados referentes ao uso do piercing e variáveis independentes foram coletados por questionário semiestruturado. A Idade média dos participantes foi 22 anos e a prevalência do piercing foi de $46,8 \%(\mathrm{n}=89)$. Houve maior prevalência no sexo feminino $49 \%$ versus masculino $19 \%(\mathrm{p}=<0,001)$. Região auricular $53,9 \%(\mathrm{n}=48)$ foi a mais utilizada. A maioria dos universitários $87,6 \%(n=78)$ inseriu o adorno em estúdios $(n=69)$ e apenas $7,9 \%(n=7)$ foram orientados a procurar um profissional de saúde para avaliação. Complicações imediatas e tardias foram experimentadas por $41,6 \%(n=37)$ e $59,5 \%(n=53)$ dos universitários, respectivamente.

PALAVRAS-CHAVE: Estudantes. Estudos Transversais. Universidade.

ÁREA TEMÁTICA: Condições Sociais e de Saúde.

\section{INTRODUÇÃO}

O piercing é uma prática que consiste em perfurar partes do corpo, para a inserção de jóias, socialmente aceito e bem popular entre os jovens. Socialmente aceito, tornou-se uma das modificações corporais mais populares entre os adolescentes e adultos jovens (LAHOUSEN et al., 2019; PRESLAR et al., 2020) sendo apreciado por ambos os sexos, com maior prevalência nas mulheres (LAHOUSEN et al., 2019; KLUEGER et al., 2019). Com o número reduzido de estudos que investiguem o uso desta jóia em universitários da área da saúde, o presente estudo teve como objetivo investigar a prevalência deste adorno em universitários da Universidade Federal dos Vales do Jequitinhonha e Mucuri, Brasil.
\end{abstract}




\section{METODOLOGIA}

Foi realizado um estudo transversal com uma amostra de 190 universitários, matriculados do primeiro ao nono período de curso da área da saúde da Universidade Federal dos Vales do Jequitinhonha e Mucuri. O curso da saúde foi selecionado por sorteio e os universitários por conveniência. $\mathrm{O}$ estudo incluiu coleta de informações por meio de um questionário semiestruturado referentes à prática do piercing e variáveis sociodemográficas como idade e sexo.

Inicialmente foi realizado um estudo piloto com 30 universitários matriculados no nono período e que não fizeram parte do estudo principal com objetivo de testar a metodologia. Algumas alterações foram feitas no questionário semiestruturado com finalidade de se obter maior clareza para o entendimento das perguntas. Observou-se ainda dificuldade em se conseguir a presença de todos os alunos, sendo decidido que as aplicações seriam realizadas em sala de aula, antes do início de suas aulas, sendo estas medidas aplicadas no estudo principal.

Foi considerado como piercing, a joia inserida em qualquer região do corpo, sendo na região auricular, pela segunda vez no pavilhão auditivo nas mulheres e no lóbulo da orelha apenas para os homens. As respostas quanto ao uso do piercing foi dicotomizada em 0 para que nunca fez uso e 1 já fez e faz uso. A aplicação dos questionários foi realizada em sala de aula, os universitários receberam os questionários codificados garantindo o sigilo e privacidade.

Os dados foram avaliados por meio do programa Statistical Package for the Social Sciences (SPSS for Windows, version 20.0, SPSS Inc, Chicago, IL, USA) e incluiu distribuição de frequência e testes de associação. O projeto foi aprovado pelo Comité de Ética em Pesquisa (parecer: 79177617.1.0000.5108).

\section{RESULTADOS E DISCUSSÕES}

Os 190 universitários avaliados, representaram aproximadamente (80\%) dos 240 alunos regularmente matriculados no curso selecionado, sendo que $20 \%$ representaram os universitários que estavam ausentes no dia da aplicação ou mesmo que se recusaram a participar.

A idade dos participantes variou entre 18 e 34 anos, com média de 22 anos. A prevalência de piercing foi de 47,3\% ( $\mathrm{n}=89)$ em adultos jovens observada neste estudo foi superior a maioria dos estudos cuja prevalência variou de 0,8\% a 42\% (CARROL et al., 2002; BROOK. et al., 2003; HENNEQUIN-HOENDERDO; SLOT VAND DER WEIJDEN, 2012). O uso do adorno foi mais prevalente no gênero feminino, sendo este achado corroborado por outros estudos (LAHOUSEN et al., 2019; STIEGER et al., 2010; KLUGER et al., 2019).

Diversos são os locais anatômicos observados para inserção do piercing, mas no presente estudo as principais regiões foram auricular $(53,9 \% \mathrm{n}=48)$, nasal, $(6,7 \% \mathrm{n}=6)$ ou ambas $(6,7 \% \mathrm{n}$ $=6$ ). Quando apenas a região do lóbulo da orelha foi considerada, houve maior prevalência no sexo feminino $49 \%$ versus masculino $19 \%(\mathrm{p}=<0,001)$. 
Quase 90\% dos universitários inseriram o piercing em estúdios, sendo relatada a utilização de técnicas de higienização e esterilização. Complicações imediatas após a colocação do piercing, tais como sangramento excessivo e dor foram experimentados por 41,6\% $(\mathrm{n}=37)$ dos universitários, e complicações tardias como inchaço, sangramento espontâneo e secreção purulenta foram observados por $59,5 \%(\mathrm{n}=53)$ dos universitários. Tal fato se reveste de maior preocupação quando percebemos que apenas 7,8\% $(\mathrm{n}=7)$ dos universitários foram orientados a procurar um profissional de saúde para avaliação e controle e apenas 37,8\% $(\mathrm{n}=34)$ terem feito alguma vez a remoção do piercing para higienização. É preocupante que a maioria dos universitários deste estudo, 77,5\% $(\mathrm{n}=69)$ inserisse seu primeiro piercing com idade inferior a 18 anos. Embora muitos estados e municípios brasileiros regulamentam e orientam a prática do piercing, a falta de uma lei nacional e escassez de cursos de formação e capacitação continuada desta profissão, possam ter contribuído para que uma alta taxa de piercing tenha ocorrido em menores de idade.

\section{CONCLUSÃO}

O estudo contribui com informações importantes sobre a prática do uso do piercing em jovens universitários da área de saúde, e os resultados evidenciam que a sua prática deve ser melhor compreendida, em razão de seus riscos à saúde e da idade precoce de ocorrência.

\section{REFERÊNCIAS}

BROOKS, T. L.; WOODS, E. R.; KNIGHT, J. R.; SHRIER, L. A. Body modification and substance use in adolescents: is there a link? Boston: Journal of Adolescent Health, 2003.

CARrol, S. T.; RIfFEnBURGH, R. H.; ROBERTS, T. A.; MYHRE, E. B. Tattoos and body piercings as indicators of adolescent risk-taking behaviors. San Diego: Pediatrics, 2002.

HENNEQUIN-HOENDERDOS, N. L.; SLOT, D. E.; VAN DER WEIJDEN, G. A. The prevalence of oral and peri-oral piercings in young adults: a systematic review. Amsterdam: International of Journal Dentistry Hygienic, 2012.

KLUGER, N.; MISERY, L.; SEITÉ, S.; TAIEB, C. Body Piercing: A National Survey in France. Paris: Dermatology, 2019.

LAHOUSEN, T.; LINDER, M. D.; GIELER, U.; HOFMEISTER, D.; TRAPP, E. M.; BORKENHAGEN, A.; KAPFHAMMER, H. P.; BRÄHLER, E. Body modification in Germany: prevalence, gender differences and attitude towards cosmetic surgery. Graz: Giornale italiano di dermatologia e venereologia. 2019.

PRESLAR, D.; BORGER, J. Body Piercing Infections. Buies Creek: StatPearls, 2019.

STIEGER, S.; PIETSCHNIG, J.; KASTNER, C. K.; VORACEK, M.; SWAMI, V. Prevalence and acceptance of tattoos and piercings: a survey of young adults from the southern German-speaking 
area of Central Europe. Viena: Percept Mot Skills, 2010. 